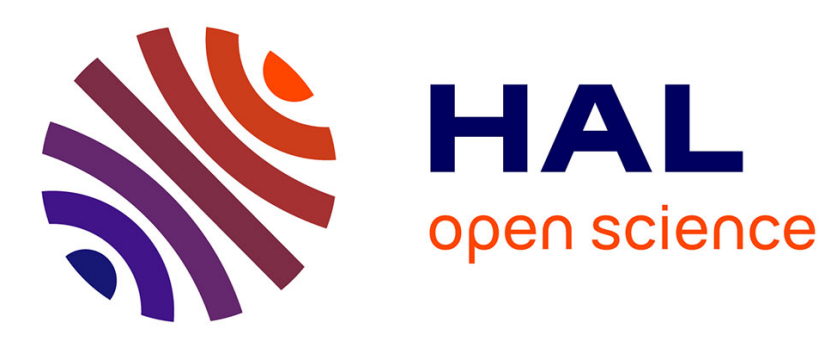

\title{
Agent-Based Modelling of Demand-Side Flexibility Adoption in Reservoir Pumping
}

Magnus Verbak, Zheng Ma, Kristoffer Christensen, Yves Demazeau, Bo Nørregaard Jørgensen

\section{- To cite this version:}

Magnus Verbak, Zheng Ma, Kristoffer Christensen, Yves Demazeau, Bo Nørregaard Jørgensen. AgentBased Modelling of Demand-Side Flexibility Adoption in Reservoir Pumping. 2019 IEEE Sciences and Humanities International Research Conference (SHIRCON), Nov 2019, Lima, Peru. pp.33-36, 10.1109/SHIRCON48091.2019.9024889 . hal-02875017

\section{HAL Id: hal-02875017 https://hal.science/hal-02875017}

Submitted on 10 Dec 2020

HAL is a multi-disciplinary open access archive for the deposit and dissemination of scientific research documents, whether they are published or not. The documents may come from teaching and research institutions in France or abroad, or from public or private research centers.
L'archive ouverte pluridisciplinaire HAL, est destinée au dépôt et à la diffusion de documents scientifiques de niveau recherche, publiés ou non, émanant des établissements d'enseignement et de recherche français ou étrangers, des laboratoires publics ou privés. 


\section{Agent-Based Modelling of Demand-Side Flexibility Adoption in Reservoir Pumping}

\author{
Magnus Værbak \\ Center for Energy Informatics \\ Morsk Mc-Kinney Møller Institute \\ University of Southern Denmark \\ Odense, Denmark \\ mavar@mmmi.sdu.dk \\ Yves Demazeau \\ Laboratoire d'Informatique de \\ Grenoble \\ Centre National de la Recherche \\ Scientifique \\ Grenoble, France \\ Yves.Demazeau@imag.fr
}

\author{
Zheng Ma \\ Center for Energy Informatics \\ Morsk Mc-Kinney Møller Institute \\ University of Southern Denmark \\ Odense, Denmark \\ zma@mmmi.sdu.dk \\ Bo Nørregaard Jørgensen \\ Center for Energy Informatics \\ Morsk Mc-Kinney Møller Institute \\ University of Southern Denmark \\ Odense, Denmark \\ bnj@mmmi.sdu.dk
}

\author{
Kristoffer Christensen \\ Center for Energy Informatics \\ Moersk Mc-Kinney Møller Institute \\ University of Southern Denmark \\ Odense, Denmark \\ kric@mmmi.sdu.dk
}

\begin{abstract}
As wind and solar power displace conventional generation in the electricity grid, there is an urgent need for technologies that can deal with the variation in supply. Demand response technology has been proposed as a solution to make the demand-side flexible and able to effectively adjust to variations in supply. However, consumers do not simply invest in demand response technologies without insurance that their investment will pay back. This paper applies agent-based simulation to investigate consumer adoption behaviour of implicit demand response solutions that allow consumers to adjust their electricity use to the hourly prices in day-ahead spot markets. The simulation uses the case study of a water supply system to show that the adoption speed of implicit demand response technology depends on both technical characteristics of the system and the business model of the implicit demand response solution provider. Hence, this paper contributes with insight on how agent-based simulation can help technology providers to design solutions which match the needs of system operators.
\end{abstract}

Keywords—agent-based modelling, demand response, water distribution, smart energy solutions, innovation adoption, simulation)

\section{INTRODUCTION}

Due to the intermittent nature of the increasing amount of wind and solar energy in the electricity grid, there is an urgent need for identifying sectors that can provide flexibility in the demand to meet the variations in the supply. Several studies have estimated the magnitude of electricity use in the water sector, and the savings that can be gained through flexibility.

Studies show that the water supply system account for up to $5 \%$ of a city's total electricity consumption whereof two thirds is used by electric pumps [1]. Furthermore, it is estimated that the water supply sector constitutes $3-4 \%$ of the total U.S. electricity consumption where $80 \%$ of electricity is used for the water pumping and transportation, including wastewater, and the remaining $20 \%$ is used for water treatment processes [2]. For the Danish water supply system, [3] assesses the potentials for load shifting to a later point in time to be $37 \mathrm{MW}$ and $7 \mathrm{MW}$ for the water supply and water treatment sectors, respectively. The potentials for load shifting to an earlier point in time are $30 \mathrm{MW}$ and $10 \mathrm{MW}$, respectively.

The water reservoir pump operators represent one of the water supply actors that can provide load shifting by adopting implicit demand response (IDR). When exposed to electricity prices that follow the day-ahead wholesale spot market, the water reservoir pump operators have the opportunity to move pump operation to hours where the electricity supply is high and/or the demand is low, and the electricity price is correspondingly cheap. However, as there are some costs imposed by switching operation solution, e.g. investments in more advanced, and thus expensive, water pumps or automatic control systems, it might not be equally viable for all operators to switch to the IDR solution.

Therefore, the following research question is proposed for this paper: "What are the characteristics of the first $50 \%$ of water reservoir pump operators to adopt implicit demand response?" To address the research question, this paper designs a simulation model to investigate the characteristics of the first $50 \%$ of a population of water reservoir pump operators who adopt IDR according to the theory of diffusion of innovation [4] (shown in Fig. 1).

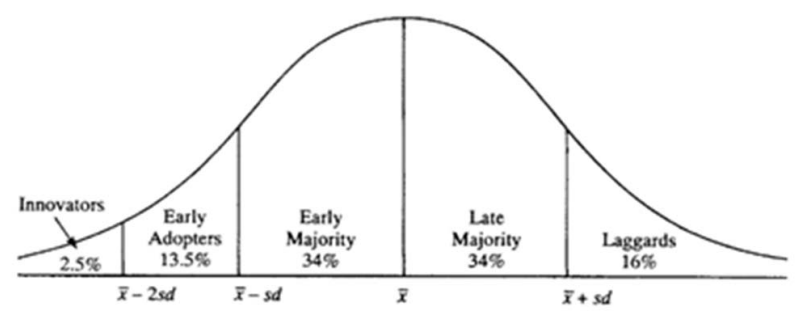

Fig. 1. Different categories of solution adopters over time [4]

The remainder of the paper is organised as follows: Sec. II gives a brief introduction to the overall structure of a water distribution system. Sec. III describes the framework of the model, including its components and interactions between these. Sec. IV contains all the inputs, assumptions and simplifications that are used for the model. Sec. V discusses the use of the model on two case studies and the results, and finally Sec. VI concludes the paper.

\section{SYSTEM DESCRIPTION}

The water distribution system (WDS), in a simple form, consists of water wells that extract water as a raw resource (from rivers, lakes, ground water, etc.), a treatment plant that renders the water drinkable and a pipe network that transfers the water from the treatment plant to end-users. Furthermore, the network includes a number of storage tanks at strategic points [5]. 
There are two types of storage tanks: the elevated tank and the clear water reservoir [6, 7]. The clear water reservoir provides emergency water reserves and buffer capacity to even out peak water consumptions at the treatment plants and allow the plants to run steadily which improves the efficiency of the treatment process. This also eliminates or reduces the need for a peak operation capacity at the treatment plant and thus reduces the nominal investment for construction of a new plant. The main purpose of the elevated tanks is to sustain and regulate pressure levels across the network, although they can be used as water storage buffers as well. However, desires to reduce the water resting time and developments in pump and automatic pressure regulation technologies have decreased the usage of elevated tanks in the Danish WDS over recent years [7].

For both types of tanks, a water pump is used to fill up the tank. According to [7], the water pumps typically run a number of subsequent hours during different periods of the day. However, for some clear water reservoirs, the main pump fills the reservoir while a secondary pump provides water directly to the consumers in hours with low electricity prices. In hours with medium prices, the main pump is switched off and water is provided from the reservoir and the secondary pump. In hours with expensive electricity prices, only the reservoir provides water and the pumps are switched off [7].

\section{SIMULATION FRAMEWORK}

This paper uses agent-based modelling, due to its ability to simulate and analyse systems where the implications of change on the overall system are difficult to predict analytically. Instead, the behaviour and goals of the individual agents in the system are defined along with the environment in which they operate. The simulation is created with the program, AnyLogic. The structure of the model contains three main modules: a decision module (DM), a domain logic module (DLM) and a business solution module (BSM), the latter which contains a submodule for each unique business solution that the stakeholders can choose between. These three modules fulfil individual roles and communicate with each other through few different message channels.

The DLM contains the representation for the physical water pumps and towers. As the DM needs to decide which of the two solutions that is the best, the DLM initiates two systems that are completely identical at the simulation start, one for each business solution. The DLM does not decide whether the pump should run in a given hour or not, as long as its limits are not exceeded. Instead, it receives a start/stop message from the BSM. However, it is still able to override the BSM's decisions if these conflict with any of the system's boundary conditions, for instance, preventing BSM from stopping the pump if the tank is almost empty or starting the pump if the tank is full. The DLM sends back the current power consumption of the pump to the respective BSM submodules which will calculate the expenses according to their individual settlement schemes. The DLM will also provide the current water levels to allow the BSM submodules to plan the operation.

The two BSM submodules continuously send their accumulated expenses to the DM. As all pumps are initially considered to run without regarding the electricity price (the standard solution), the DM decides when the pump operator will adopt the IDR solution. In this paper, the adoption occurs as soon as the accumulated costs of the standard solution exceeds those that would have been imposed by using the IDR solution in the same period of time (in this case from the beginning of the simulation) plus the costs of switching between solutions, i.e. the condition in Eq. 1 is true.

$$
C_{\text {acc.standard }} \geq C_{\text {acc.IDR }}+C_{i n v}
$$

Here, $C_{\text {acc.standard }}$ denotes the accumulated electricity costs for the standard solution, $C_{a c c . I D R}$ denotes the accumulated electricity costs for the IDR solution, and $C_{i n v}$ denotes the cost of switching to the IDR solution. The DM therefore takes a retro-perspective approach to determine the return of investment time for adoption of the IDR solution.

When a population member decides to adopt the IDR solution, it is added to the "adopted" group. When $50 \%$ of the population have moved to this group, the simulation is stopped, and the elapsed period of time is noted.

\section{EXPERIMENTAL DESIGN}

The simulation model makes several assumptions, partly to simplify the model and partly to represent a generic WDS. Assumptions for the overall model and the individual modules along with general input data and conditions are given separately in the following subsections.

\section{A. Assumptions}

No taxes and tariffs are added to the electricity prices. The price of adopting the IDR solution is set as the cost of purchasing a pump with a frequency transformer and a control system compared to the cost of a pump without any possibility for control, except for simple on/off regulation. To get an impression of this cost difference, two Grundfos pumps models that approx. match the requirements of the systems simulated in this paper are identified by using the Grundfos Product Center dimensioning tool [8]. The C 32-3 and CRE 15-3 models were chosen for the simple pump and regulated pump, respectively. With respective prices of $28,820 \mathrm{DKK}$ and 32,295 DKK (the prices only include the pump components), an estimated investment cost is set to 3500 DKK. The population size is set to 100 , and the simulation starts at the beginning of 2017 and ends when $50 \%$ of the pumps have adopted the IDR solution or by the end of 2026 (after ten years), whichever comes first. Wholesale spot market prices are available for 2017 and 2018, and these prices are repeated for every two years passed.

Currently, the pump operator is the only stakeholder represented in the model. Other agents affect the pump operator, including the water consumers, the Nord Pool Spot Market and the distribution system operator (DSO)/ electricity retailer, as they define the water consumption pattern and electricity pricing, respectively. However, these, in turn, are not affected by any conditions in the model and are therefore not considered stakeholders within the scope of the experiment. This will likely change as additional layers of complexity are added to the model as part of future research.

\section{B. Domain Logic}

To capture differences in the sizes of customer bases that a real population of water reservoirs serve, each member of the population is assigned a basis hourly water consumption rate according to a truncated normal distribution with min./max. interval $5.5-12 \mathrm{~m}^{3} / \mathrm{h}$, mean $8.75 \mathrm{~m}^{3} / \mathrm{h}$ and spread 4 . This interval is based on the hourly peak loads given in [9]. As these values are peak loads and the maximum peak load 
factor is 2 (see Table I), the values from [9] are divided by 2 . The water consumption rates apply for 1-hour blocks according to the schedule shown in Table I. The schedule contains load factors at different times of the day, e.g. a peak load of 1.5 times the basis hourly load between 09:00 and 15:00 on weekdays. Note that the water consumption schedule is based on a mix of the hourly load profiles in [9] to represent a generic WDS where the consumer composition includes a variety of different consumer types, e.g. apartments, holiday homes, institutions, commercial buildings, etc. Further literature on water consumption patterns and predictions can be found in [10]. Monthly variations in the water consumption are omitted from this study as these are mostly apparent in systems with a significant number of holiday homes [9].

The size of the water reservoir is set to the highest daily consumption that is expected to occur during the entire simulation, and it has no upper boundary on the emptying rate. There is no leakage of water, nor is there any downtime for maintained or failure on components in the system. Furthermore, even though they are crucial for the operation of a real pump on a WDS, pressure and pipe capacity aspects of the network are considered beyond the scope of this study.

The pumps are characterised by two parameters: the pump rate and the power rate. In order to avoid situations where a reservoir is empty and the water demand outpaces the pump rate, the rate is set equal to the highest expected hourly water demand. On top of this, an extra capacity is added that follows a truncated normal distribution with $\mathrm{min}$./max. interval 0-20 $\%$ of the initial capacity, mean $10 \%$ and spread 1 . This extra capacity serves to investigate the pumping capacity's effect on the adoption rate. When on, the pumps always operate at their given rates. A pump's power rating is found using Eq. (2) [5].

$$
P_{\text {pump }}=\frac{\rho_{\text {water } \cdot g \cdot h \cdot \dot{V}}}{\mu}
$$

Here, $h$ denotes the required pressure head, $V^{*}$ denotes the pump rate, and $\mu$ denotes the shaft efficiency of the pump. Both the pressure head and the efficiency are set to be constant, even though they vary in real systems depending on the amount of water present in the reservoirs and the pressure on other parts of the WDS [11]. They are set according to an example in [11], using min./max. interval 68.2-75.1\%, mean $71.7 \%$ and spread 1 for the efficiency and min./max. interval 36.6-42.9 m, mean $39.8 \mathrm{~m}$ and spread 1. Note that the efficiency and pressure head values for real water reservoirs might be significantly different, however the purpose here is to test their effects on the adoption rate.

TABLE I. WATER CONSUMPTION SCHEDULES FOR WEEKDAYS AND WEEKENDS

\begin{tabular}{|c|c|c|c|c|c|}
\hline \multicolumn{3}{|c|}{ Weekdays } & \multicolumn{3}{c|}{ Weekends } \\
\hline Load factor & $\begin{array}{l}\text { Start } \\
\text { time }\end{array}$ & $\begin{array}{l}\text { End } \\
\text { time }\end{array}$ & Load factor & $\begin{array}{c}\text { Start } \\
\text { time }\end{array}$ & $\begin{array}{c}\text { End } \\
\text { time }\end{array}$ \\
\hline 0.5 & $00: 00$ & $05: 00$ & 0.5 & $00: 00$ & $07: 00$ \\
\hline 1.0 & $05: 00$ & $07: 00$ & 1.5 & $07: 00$ & $14: 00$ \\
\hline 2.0 & $07: 00$ & $09: 00$ & 1.0 & $14: 00$ & $22: 00$ \\
\hline 1.5 & $09: 00$ & $17: 00$ & 0.5 & $22: 00$ & $00: 00$ \\
\hline 2.0 & $17: 00$ & $20: 00$ & & & \\
\hline 1.0 & $20: 00$ & $00: 00$ & & & \\
\hline
\end{tabular}

Finally, the pumps have no limits on the number of consecutive hours they can operate, nor do they have any on/off cooldown before they can be turned off/on.

\section{Solution Design}

\section{1) Standard Solution}

The standard business solution represents the operation of the water pump without IDR. The standard business solution submodule (StandBSM) receives messages containing the current water level from the LDM, and if the water level diminishes below $25 \%$ of the tank capacity the StandBSM sends a start message to the DLM. The pump then operates continuously until the water level has reached $75 \%$ of the tank capacity after which the StandBSM sends a stop signal.

\section{2) IDR Solution}

The IDR business solution employs the variations in the wholesale spot market price to reduce the electricity bill. At the beginning of each 24-hour cycle, which happens at 15:00 each day, the wholesale spot market prices for the next 24 hours become available and a forecast of the total water consumption for the next day is made as well.

With the above information being available, the IDR business solution submodule (IDRSBM) schedules the cheapest $N$ hours for pump operation. The number of hours is set to ensure that the water levels at the beginning and the end of each 24 hours cycle 15:00-to-15:00 are identical. When the cycle starts, sometimes a production scheduled hour is encountered where the tank is full, and pumping is not possible. If that is the case, the pump will try to replace the water that is eventually consumed during the hour by starting operation sometime in the middle of the hour and until its end. The cancelled operation is then rescheduled for a later hour of the cycle (this is always possible due to the pump rate being larger than the max. hourly water consumption rate). For instance, if the reservoir is full at the beginning of an hour that is scheduled for water production and the water consumption rate for the given hour is $2 \mathrm{~m}^{3} / \mathrm{h}$ while the pumping rate is 5 $\mathrm{m}^{3} / \mathrm{h}$, the pump operates during the last $24 \mathrm{~min}$. of the hour, thus reaching a full reservoir at the end of the hour. The remaining $3 \mathrm{~m}^{3}$, corresponding to $36 \mathrm{~min}$. of operation, are then moved to the $N^{\text {th }}$ hour on the schedule or $N^{\text {th }}+1$ hour, if there are not enough "vacant minutes" in the $N^{\text {th }}$ hour.

\section{V.CASE STUDY}

This paper investigates two case studies by using the designed simulation model: 1), the reservoir capacity is dimensioned after the highest daily water consumption and starts out being full, and 2), the reservoir capacity is doubled in size while starting with the same amount of water, thus being initially half full. All other parameters are identical for the two case studies.

For the initially full tank scenario, the first adopters (or "innovators") act by the end of 2020, after which the bulk adopt until the $50 \%$ mark is reached by the $6^{\text {th }}$ of December 2023, as shown in Fig. 2. Continuing the simulation beyond the $50 \%$ mark reveals that the adoption share after ten years is $79 \%$. The adoption rate is significantly higher when the tank size is doubled while the initial water level is unchanged. Here, the $50 \%$ mark occurs almost two and a half years earlier, at the $25^{\text {th }}$ of August 2021, as shown in Fig. 3 (the 100 $\%$ mark occurs at the $11^{\text {th }}$ September 2026, just before the ten years have passed). The double-sized tank case gets a faster adoption rate, because the water level can both increase and 
decrease from the daily starting water level. In the case study with the full tank, the water can only decrease from the starting level, which impairs the opportunities for moving operation to earlier hours of a 24 hours cycle.

The average characteristics of the adopters in the two figures show that the larger pumps have a faster adoption rate compared to the smaller ones, as the average pump rate of the adopted population gradually decreases. The same result goes for the pumps connected to reservoirs with relatively large water demands, albeit to a less extent with the full tank scenario. Meanwhile, the pump efficiency/pumping head ratio (see Eq. (2)) does not seem to have a strong impact on the adoption willingness as its average fluctuates up and down with new adopters. This might be explained by the relatively small variances of the efficiency and pump head parameters compared to the water demand and pumping rate variances. In relation to this, it should be noted that it is hard to isolate the effects of the individual parameters in this case study due to their intercorrelations caused by the conditions described in section IV.B and in particular Eq. (2).
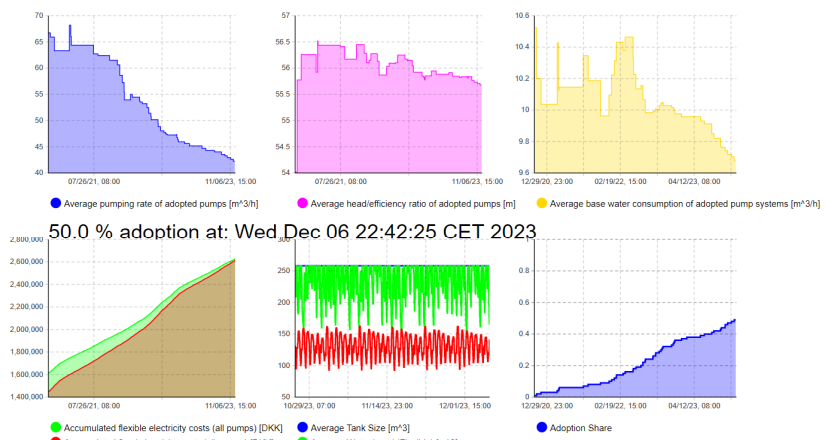$$
\text { - Accoum }
$$

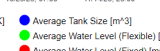

The top graphs denote the development of certain parameter averages for adopted pumps, as more and more pumps adopt. The bottom left graph depicts the accumulated costs of the two solutions, aggregated over the population. The bottom middle graph shows the average water levels over the population for the two solutions. The bottom right graph shows the adoption share development.

Fig. 2. The results at the $50 \%$ adoption share point with initially full tank
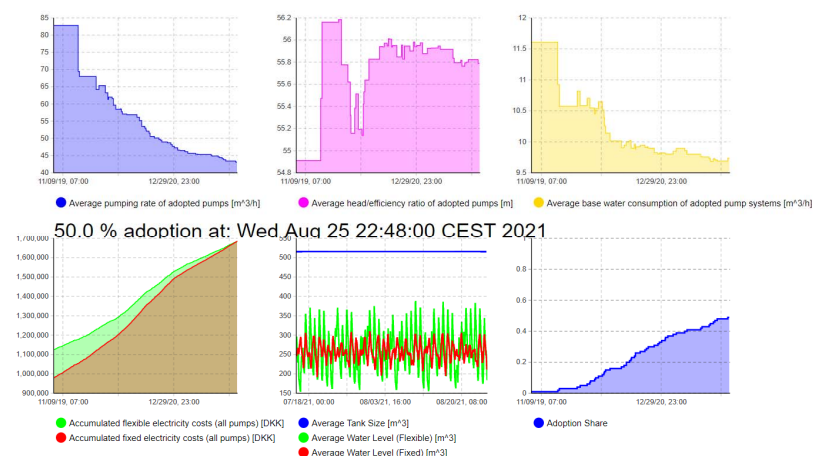

Fig. 3. The results at the moment of $50 \%$ adoption share with double-sized tank, initially half full

The model comes with multiple limitations. The, perhaps, two most significant of those are as follows:

The parameters used in this paper are based on generic WDS's and real systems might therefore differ significantly from the ones presented in this paper. When for a real WDS population has been gathered it should, however, be simple to insert these values into the model.
The limited scope of the model is more significant, as the operation of the water pumps is not only affected by other actors on the electricity and WDS business ecosystems but also affects those actors in return. Integrating these other actors into the model is therefore necessary if the simulation model is to give a realistic picture of a water tower pump operation. Examples of these additional actors might include pump technology suppliers, water treatment plants, DSO's, electricity retailers and balance responsible parties. For instance, the DSO is a very crucial beneficiary of the IDR adoption by the pump operators. With peak load shaving, the need for future extensions and maintenance to the grid, and thus expenses paid by the DSO, will decrease.

\section{CONCLUSION}

This paper develops an agent-based simulation model to investigate how the initial design and operational pattern of water tanks in domestic water systems influence the adoption rate of implicit demand response solutions in water reservoir pump operation that exploit the hourly electricity price variations in the day-ahead wholesale electricity spot markets. The simulation results show that shifting from the standard solution that does not adjust pump operation to the whole-sale electricity prices to the implicit demand response solution is more attractive when the initial water level is half of the tank capacity, as this scenario reaches a $50 \%$ adoption share almost two and a half years earlier than the full-tank scenario. Furthermore, the results show that large pumps and domestic water systems with higher demands adopt earlier. Finally, this paper demonstrates how agent-based simulation can help technology providers and adopters to evaluate the value proposition of new innovations.

\section{REFERENCES}

C. Mkireb, A. Dembele, A. Jouglet, and T. Denoeux, "Robust Optimization of Demand Response Power Bids for Drinking Water Systems," Applied Energy, vol. 238, pp. 1036-1047, Mar 2019.

K. Oikonomou, M. Parvania, and S. Burian, "Integrating water distribution energy flexibility in power systems operation," in 2017 IEEE Power \& Energy Society General Meeting, 2017, pp. $1-5$.

H. C. Gils, "Assessment of the theoretical demand response potential in Europe," Energy, vol. 67, no. C, pp. 1-18, 2014. M. E. Rogers, 5th, Ed. Diffusion of Innovations. Free Press, 2003.

A. H. Kristensen, "Ledningsnet," in Vandforsyning, E. Karlsen and S. Inga, Eds. 3rd ed.: Nyt Teknisk Forlag, 2014, pp. 681734.

V. Bhardwaj, "Reservoirs, Towers, and Tanks," in Drinking Water Storage Facilities vol. Tech Brief, ed: National Drinking Water Clearinghouse at West Virginia University.

S. Schmidt, "Beholderanlæg," in Vandforsyning, E. S. Karlsen, Inga Ed. 3rd ed.: Nyt Teknisk Forlag, 2014, pp. 619-640. Grundfos Product Center [Online]. Available: https://productselection. grundfos.com/front-

page.html? pumpsystemid $=664556790 \&$ qcid $=664560392$

A. H. Kristensen, "Vandforbrug," in Vandforsyning, E. Karlsen and S. Inga, Eds. 3rd ed.: Nyt Teknisk Forlag, 2014, pp. 321344.

C. Mkireb, A. Dembélé, A. Jouglet, and T. Denoeux, "Robust Optimization of Demand Response Power Bids for Drinking Water Systems," Applied Energy, vol. 238, pp. 1036-1047, 2019/03/15/ 2019.

K. Ellemose, "Pumpeanlæg," in Vandforsyning, E. Karlsen and S. Inga, Eds. 3rd ed.: Nyt Teknisk Forlag, 2014, pp. 643-658. 